University of Wollongong

Research Online

Faculty of Engineering and Information

Faculty of Engineering and Information

Sciences - Papers: Part A

Sciences

$1-1-2007$

Performance comparison of subtractive resistive readout with conventional resistive readout for a high-resolution compact gamma camera

Y J. Qi

Chinese Academy of Sciences, yujin@uow.edu.au

M J. Zhang

Chinese Academy Of Sciences

C L. Zhao

Chinese Academy Of Sciences

R F. Wojcik

Ray Vision Inc

Follow this and additional works at: https://ro.uow.edu.au/eispapers

Part of the Engineering Commons, and the Science and Technology Studies Commons

Research Online is the open access institutional repository for the University of Wollongong. For further information contact the UOW Library: research-pubs@uow.edu.au 


\title{
Performance comparison of subtractive resistive readout with conventional resistive readout for a high-resolution compact gamma camera
}

\begin{abstract}
The purpose of this study was to investigate an optimal readout for a high-resolution compact gamma camera with maximum performance in crystal element identifications. The compact camera is based on a pixellated $\mathrm{Nal}(\mathrm{TI})$ crystal with $1.2 \mathrm{~mm}$ pixel size coupled to a 5" Hamamatsu R3292 PSPMT. A conventional resistive-chain readout was initially developed for the camera. Then a novel subtractive resistive readout developed was utilized to optimize the performance of the camera. The performance of the camera was evaluated by raw flood images of a 137Cs source. The results show that the conventional resistive readout results in a significant shrinkage of the useful field-of-view (UFOV) of the detector with a maximum of resolvable crystal elements of 64 times 64 , which is about $89 \%$ of the active-area of the PSPMT's $(\sim 10 \mathrm{~cm})$. The subtractive resistive readout can maximize the crystal element identifications up to 71 times 71 while improve the UFOV of the detector up to almost the full active- area of the PSPMT's. In the central region of the camera, the subtractive resistive readout also improve the peak-valley ratio of the crystal elements from 1.5:1 to $2: 1$ as compared the conventional resistive readout. The phantom and in vivo mouse imaging studies demonstrate that the compact camera with subtractive resistive readout can provide very good performance for high-resolution SPECT. We concluded that the subtractive resistive readout is an effective approach for an optimal readout for the development of high-resolution compact gamma cameras.
\end{abstract}

\section{Keywords}

comparison, performance, resolution, subtractive, camera, resistive, readout, gamma, conventional, compact, high

\section{Disciplines \\ Engineering | Science and Technology Studies}

\section{Publication Details}

Qi, Y. J., Zhang, M. J., Zhao, C. L. \& Wojcik, R. F. (2007). Performance comparison of subtractive resistive readout with conventional resistive readout for a high-resolution compact gamma camera. 2007 IEEE Nuclear Science Symposium Conference Record (NSS) (pp. 3725-3728). USA: IEEE. 


\title{
Performance Comparison of Subtractive Resistive Readout with Conventional Resistive Readout for a High-resolution Compact Gamma Camera
}

\author{
Y. J. Qi, Member, IEEE, M. J. Zhang, C. L. Zhao, R. F. Wojcik
}

\begin{abstract}
The purpose of this study was to investigate an optimal readout for a high-resolution compact gamma camera with maximum performance in crystal element identifications. The compact camera is based on a pixellated NaI(TI) crystal with $1.2 \mathrm{~mm}$ pixel size coupled to a 5" Hamamatsu R3292 PSPMT. A conventional resistive-chain readout was initially developed for the camera. Then a novel subtractive resistive readout developed was utilized to optimize the performance of the camera. The performance of the camera was evaluated by raw flood images of a ${ }^{137} \mathrm{Cs}$ source. The results show that the conventional resistive readout results in a significant shrinkage of the useful field-ofview (UFOV) of the detector with a maximum of resolvable crystal elements of $64 \times 64$, which is about $89 \%$ of the active-area of the PSPMT's $(\sim 10 \mathrm{~cm})$. The subtractive resistive readout can maximize the crystal element identifications up to $71 \times 71$ while improve the UFOV of the detector up to almost the full activearea of the PSPMT's. In the central region of the camera, the subtractive resistive readout also improve the peak-valley ratio of the crystal elements from 1.5:1 to $2: 1$ as compared the conventional resistive readout. The phantom and in vivo mouse imaging studies demonstrate that the compact camera with subtractive resistive readout can provide very good performance for high-resolution SPECT. We concluded that the subtractive resistive readout is an effective approach for an optimal readout for the development of high-resolution compact gamma cameras.
\end{abstract}

\section{INTRODUCTION}

$\mathrm{I}_{\mathrm{r} e}^{\mathrm{N}}$ $\mathrm{N}$ recent years there have been many efforts to develop highresolution compact gamma cameras for nuclear medicine and animal research applications[1-6]. In terms of size, cost effectiveness and performance, the use of a pixellated scintillator crystal array coupled to a position sensitive photomultiplier tube (PSPMT) still remains the best choice for high-resolution compact gamma cameras. The design and implementation of optimal readout electronics for the multianode PSPMT are very essential for the compact camera to achieve a maximized performance.

There are commonly two readout schemes for the gamma camera based on the multi-anode PSPMT. One is the conventional resistive-chain readout[7-8]. Although offering some advantage such as simplicity and the reduction of the

Manuscript received November 22, 2007. This work was supported in part by the Chinese Nature Science Foundation under Grant number 30570520 and the Shanghai Pujiang Program.

Y.J. Qi, M.J. Zhang, C.L. Zhao are with the Shanghai Institute of Applied Physics, Chinese Academy of Sciences, Shanghai 201800, China (telephone: 86-21-59554664, e-mail: yujinqi@yahoo.com).

R. F. Wojcik is with RayVision Inc., Yorktown, VA 23693, USA number of readout channels, it significantly reduces the performance of the camera in the spatial resolution and image quality due to its poor signal-to-noise ratio. Another is the individual channel readout[9-10]. Although it could provide the best performance for the camera, the complexity of the readout electronics and the expense for the data process and acquisition requirements would be significantly increased as the readout channels increase. So there have been many efforts to develop an optimized readout which maximizes the performance of the compact camera while keeping the simplicity of the conventional resistive readout. Recently the detector group of Jefferson National Laboratory has successfully developed such a readout called subtractive resistive readout to achieve the purpose[11-12].

In this study we investigate an optimal readout for our compact gamma camera based on a pixellated $\mathrm{NaI}(\mathrm{Tl})$ crystal array coupled to a 5" Hamamatsu PSPMT tube. The detector performances for both conventional resistive readout and subtractive resistive readout have been evaluated in the flood images of the camera using a ${ }^{137} \mathrm{Cs}$ source. Then an optimal readout for our camera would be determined basing on their performances.

\section{MATERIALS AND METHODS}

\section{A. Compact gamma camera}

The compact gamma camera composed of a pixellated $\mathrm{NaI}(\mathrm{Tl})$ crystal array and a 5" diameter Hamamatsu R3292 position sensitive photomultiplier tube (PSPMT). The crystal array from the Saint Gobain crystals and detectors was $112 \mathrm{~mm}$ square with $1.2 \mathrm{~mm} \times 1.2 \mathrm{~mm}$ pixel elements, $1.4 \mathrm{~mm}$ pixel pitch and $5 \mathrm{~mm}$ crystal thickness. The crystal array has $80 \times 80$ pixels and is encapsulated in a compact housing with a $2 \mathrm{~mm}$ thick quartz window. The light output from the scintillator is detected by the directly coupled PSPMT. The R3292 is a cross-wire anode PSPMT which has $28 \mathrm{X}$ and $28 \mathrm{Y}$ anode wires and a minimum photocathode size of $100 \mathrm{~mm}$ in diameter. The 12 stages of parallel mesh dynode structure result in a gain factor of $10^{5}$.

\section{B. Conventional resistive readout}

The conventional resistive readout was developed basing on the design shown in the Hamamatsu R3292 manual. A sketch diagram of the readout scheme is shown in Fig.1. The anode wires in each $\mathrm{X}$ and $\mathrm{Y}$ direction are chained together by $1 \mathrm{~K}$ resistors and lead out four outputs. The four output signals 
were amplified and shaped by 4 low-noise charge-sensitive preamplifiers and then output to the data acquisition system. The standard center-of-gravity (also be called Anger Logic) method was used to calculate the position of the incident gamma-ray.

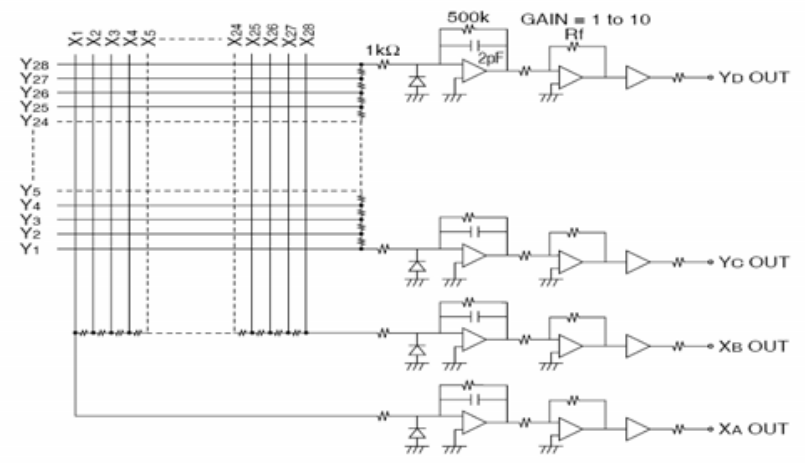

Fig.1 Sketch diagram of conventional resistive readout.

\section{Subtractive resistive readout}

The novel subtractive resistive readout was developed by Ray Visions Inc. The basic idea of the subtractive resistive readout scheme is to implement a fractional subtraction technique in the readout circuitry board to subtract the long tail of the charge distribution induced on the anode plane of the PSPMT, then a truncated center-of-gravity (COG) algorithm is applied to improve the accuracy of the position determination as well as the useful field-of-view of the camera.

The simplified diagram of the subtractive resistive readout is shown in Fig. 2. It consists in a pre-amplification circuit, a selection circuit of local region and a resistive-chain readout circuit. Like the conventional resistive-chain readout, the subtractive resistive readout also produce 4 output signals to the data acquisition system.

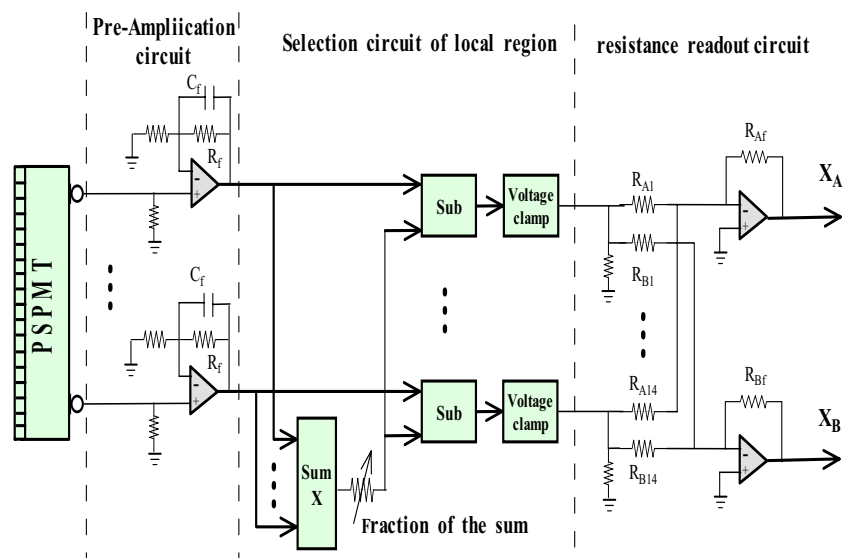

Fig.2 A simplified diagram of the subtractive resistive readout in $\mathrm{X}$ direction of the PSPMT.

We used the same data acquisition system for both readout circuitry boards. The sum of the four signals was also used to provide the event trigger of the DAQ system. The data acquisition system is based on a 12-bit PCI-6110E ADC card from the National Instrument Inc. The DAQ software was based on the Kmax from the Sparrow Inc. The performances of both the conventional resistive readout and the subtractive resistive readout were tested in the flood images using a ${ }^{137} \mathrm{Cs}$ source with gamma energy of $637 \mathrm{keV}$.

\section{RESULTS}

\section{A. Crystal element identifications}

The raw flood images acquired with the conventional resistive readout and the subtractive resistive readout using a ${ }^{137}$ Cs source are shown in Fig.3. The results show that the conventional resistive-chain readout results in a significant shrinkage of the useful field-of-view (UFOV) of the detector as compared to the subtractive resistive readout. The conventional resistive readout is barely able to distinguish a maximum of $64 \times 64$ crystal elements while the subtractive resistive readout could resolve a maximum of $71 \times 71$ crystal elements. The useful field-of-view (FOV) of the camera with the conventional resistive-chain readout is only $89.6 \mathrm{~mm}$
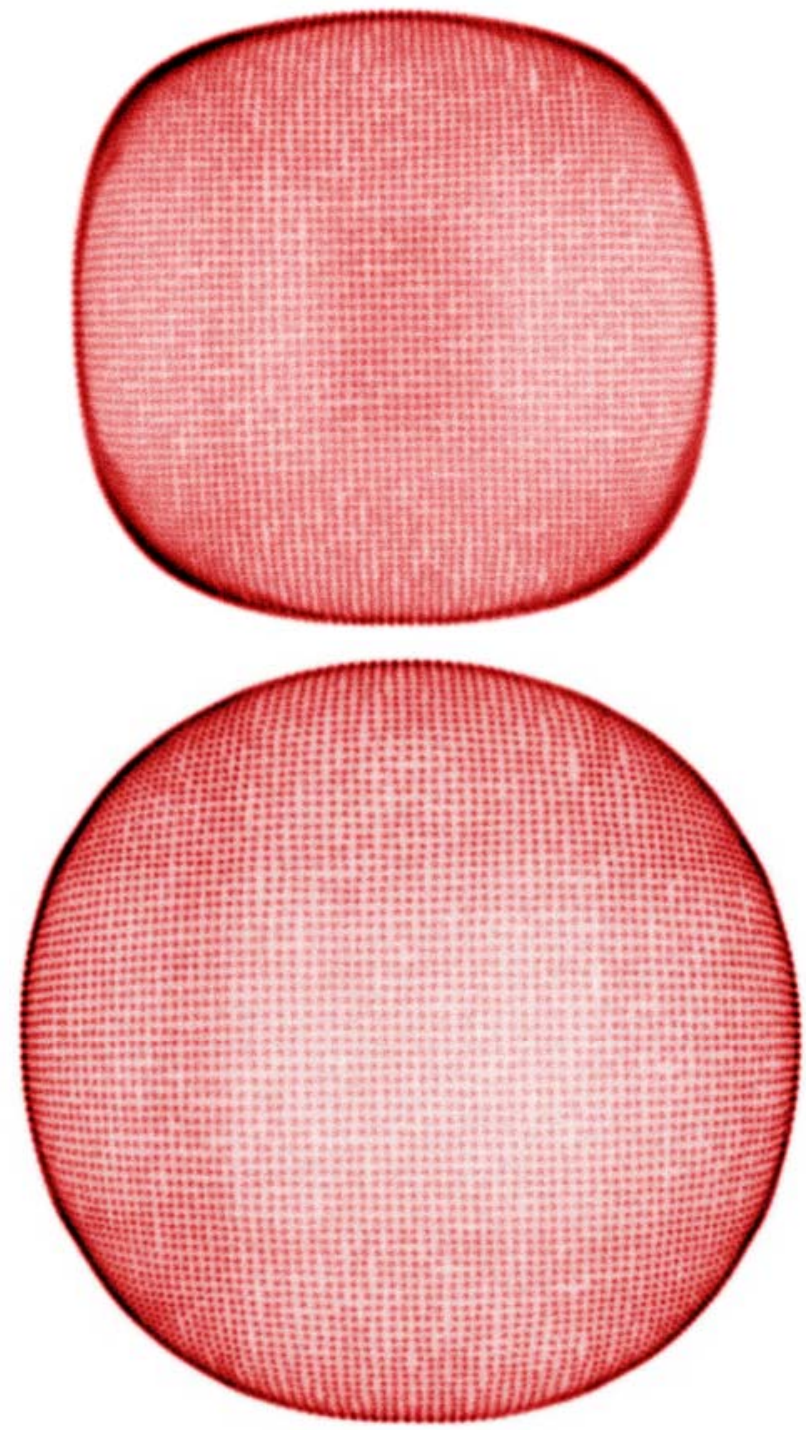

Fig. 3 Comparison of the raw flood images obtained with conventional resistive readout (top) and subtractive resistive readout(bottom) in resolving the $1.2 \times 1.2 \times 5 \mathrm{~mm} \mathrm{NaI(Tl)} \mathrm{crystal} \mathrm{array}$ with 5" Hamamatsu R3292 PSPMT. Both images were obtained using a ${ }^{137} \mathrm{Cs}$ source and plotted in the same scale. 
which is about $89 \%$ of the active area of the PSPMT $(\sim 100 \mathrm{~mm})$ while the subtractive resistive readout expands the useful FOV to reach the full active area of the PSPMT.
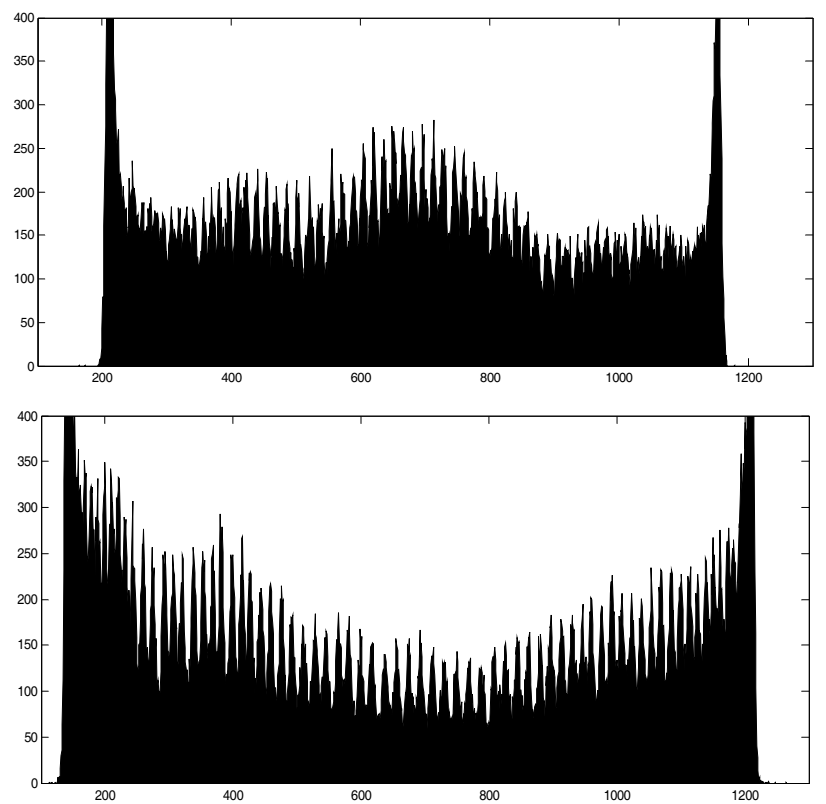

Fig. 4 The profile histograms of the middle pixel row of the raw flood images: (top) from the conventional resistive-chain readout and (bottom) from the subtractive resistive readout.

The profile histograms of the selected rows in the central region of the raw flood images are shown in Fig. 4. We can see a large improvement of the peak-to-valley ratio in the crystal element separations. In the central region, the subtractive resistive readout improves the peak-to-valley ratio from 1.5:1 to $2: 1$ as compared the conventional readout.

Using the subtractive resistive readout, the compact gamma camera was calibrated with ${ }^{99 \mathrm{~m}} \mathrm{Tc}$ and ${ }^{125} \mathrm{I}$ sources. The

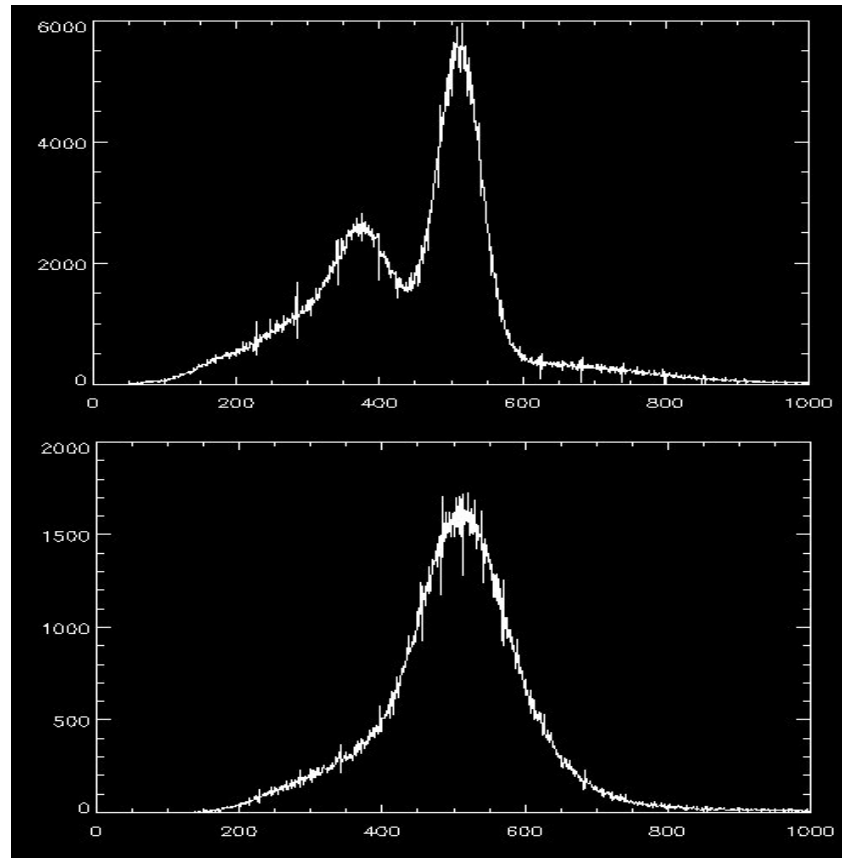

Fig. 5 The measured energy spectra obtained from the compact gamma camera with the subtractive resistive readout using ${ }^{99 \mathrm{~m}} \mathrm{Tc}$ (top panel) and ${ }^{125} \mathrm{I}$ (bottom panel) sources. measured global energy spectra are shown in Fig. 5. The energy resolutions of the compact gamma camera with the subtractive resistive readout are $\sim 16 \%$ and $\sim 25 \%$ for the gamma-ray at energies of $140 \mathrm{keV}$ and $\sim 30 \mathrm{keV}$, respectively.

\section{B. Phantom and small animal imaging tests}

The compact gamma camera with the subtractive resistive readout fitted with a pinhole collimator has been used for small animal SPECT imaging. Phantom scans were performed to evaluate the system performance in spatial resolution. Fig. 6 shows reconstructed transaxial image of an ultra micro-Deluxe phantom with hot-rod insert using a $0.6 \mathrm{~mm}$ pinhole aperture. Rod diameters are equal to the spacing between rods, with diameter of $0.75,1.0,1.35,1.7$, $2.0,2.4 \mathrm{~mm}$ for the six sectors. The phantom was filled with $\sim 4 \mathrm{mCi}{ }^{99 \mathrm{~m}} \mathrm{Tc}$-pertechnetate in water solution and a total of 90 projections were acquired over $360^{\circ}$ and 30 seconds per view at a radius-of-rotation of $2.5 \mathrm{~cm}$ with a magnification of 4 . The $1.0 \mathrm{~mm}$ sector of the phantom is clearly resolved, and the

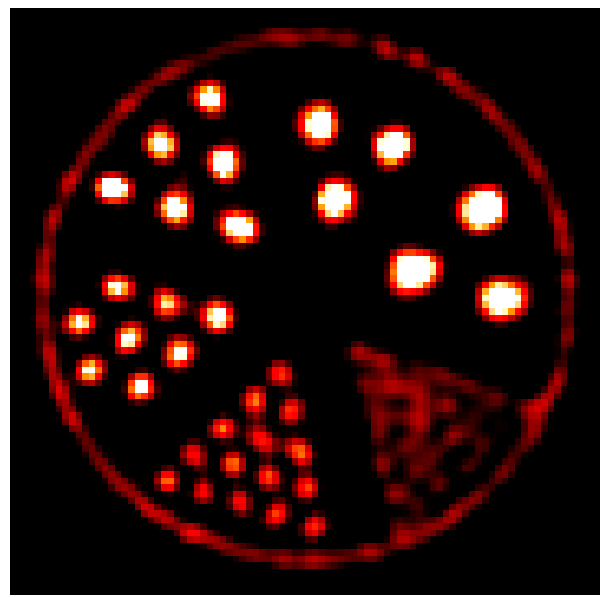

Fig. 6 Reconstructed transaxial images of an ultra micro-Deluxe phantom with hot rods insert obtained from the compact gamma camera with subtractive resistive readout using $0.6 \mathrm{~mm}$ pinhole aperture. The phantom was filled with $\sim 4 \mathrm{mCi}{ }^{99 \mathrm{~m}} \mathrm{Tc}$-pertechnetate in water solution. The rod diameters are $0.75,1.0,1.35,1.7,2.0$ and $2.4 \mathrm{~mm}$, respectively.

$0.75 \mathrm{~mm}$ sector is partially resolved.

In vivo animal experiments were performed. Fig. 7 shows

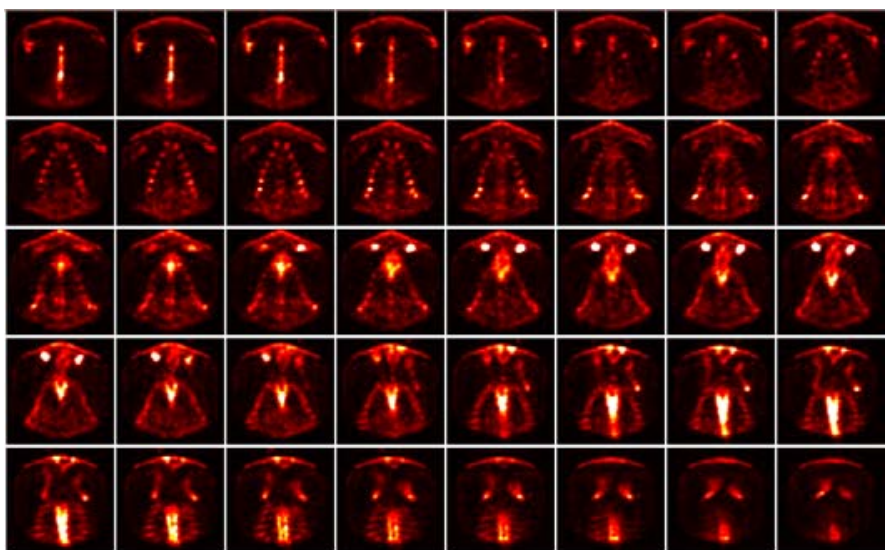

Fig. 7 Coronal slice images through the chest of a $30 \mathrm{~g}$ normal mouse injected with $\sim 5 \mathrm{mCi}{ }^{99 \mathrm{~m}} \mathrm{Tc}-\mathrm{MDP}$ obtained from the compact gamma camera with subtractive resistive readout using a pinhole collimator with a $1.0 \mathrm{~mm}$ pinhole aperture. The images were reconstructed using a 3D pinhole OSEM algorithm at 8 iterations and 10 subsets with $(0.25 \mathrm{~mm})^{3}$ voxel size 
the reconstructed coronal slice images through the chest of a $\sim 30$-gram normal mouse which was injected $\sim 5 \mathrm{mCi}{ }^{99 \mathrm{~m}} \mathrm{Tc}$ MDP. The bone scan imaging was acquired with a total of 90 projection views over $360^{\circ}$ and 30 seconds per view using a $1.0 \mathrm{~mm}$ pinhole collimator. The images were reconstructed using a $3 \mathrm{D}$ pinhole OSEM algorithm at 8 iterations and 10 subsets with $(0.25 \mathrm{~mm})^{3}$ voxel size. High spatial resolution is evident in the bone scan images, where small bone structure such as ribs are well resolved. The results demonstrate that the compact gamma camera based on the subtractive resistive readout is reliable for high quality small animal SPECT imaging.

\section{CONCLUSION AND DISCUSSION}

We have investigated an optimal readout for a highresolution compact gamma camera. The performances with conventional resistive-chain readout and subtractive resistive readout are compared in the raw flood images in terms of the ability of the crystal element identifications. The subtractive resistive readout shows significant advantages in maximizing the crystal element identifications and improving useful FOV as compared to the conventional resistive-chain readout. The phantom and mouse imaging studies demonstrate that the compact camera with subtractive resistive readout can provide very good performance for high-resolution pinhole SPECT.

In conclusion, the subtractive resistive readout is an effective approach for optimal readout electronics for the development of high-resolution compact gamma cameras. The subtractive resistive readout could be used for the readout electronics of the newly multi-anode PSPMT with much higher integration of readout channels.

\section{REFERENCES}

[1] R.Wojcik, S. Majewski, D. Steinbach and A.G. Weisenberger, "High Spatial resolution Gamma Imaging Detector Based on 5" Diameter R3292 Hamamatsu PSPMT," IEEE Trans. Nucl. Sci., vol. 45, no.3, pp.487-491, June, 1998.

[2] N. Schramm, A. Wirrwar, F. Sonnenberg, and H. Halling, "Compact high resolution detector for small animal SPECT," IEEE Trans. Nucl. Sci., vol. 47, pp. 1163-1167, June, 2000.

[3] D.P. McElroy, L.R. MacDonald, F.J. Beekman, Y. Wang, B.E. Patt, J.S. Iwanczyk, B.M.W. Tsui, and E.J. Hoffman, "Performance evaluation of A-SPECT: A high-resolution desktop pinhole SPECT system for imaging small animals," IEEE Trans. Nucl. Sci., vol. 49, no. 5, pp. 21392147, 2002.

[4] T.E. Peterson, H. Kim, M.J. Crawford, B.M. Cershman, W.C.J. Hunter, H.B. Barber, L.R. Furenlid, D.W. Wilson, J.M. Woolfenden, and H.H. Barratte, "SemiSPECT: A small-animal imaging system based on eight CdZnTe pixel detectors," 2002 IEEE NSS-MIC Conference Record, Norfolk, Nov.10-16, 2002.

[5] R. Pani, R. Pellegrini, M.N. Cinti, C. Trotta, G. Trotta, R. Scafe, M. Betti, F. Cusanno, L. Montani, G. Iurlaro, F. Garbaldi, Del A. Guerra, "A novel comapct gamma camera based on flat panel PMT," Nucl. Instr. Meth. Phys. Res A, vol. 513, no.1, pp36-41, 2003.

[6] Y. Qi, B.M.W. Tsui, Y. Wang, B. Yoder, A. Weisenberger, R. Wojcik and S. Majewski, "Development and characterization of a highresolution microSPECT system for small animal imaging”, SmallAnimal SPECT Imaging, M.A. Kupinski and H.H. Barrett, Eds. New York: Springer, Chapter 21, 2005, pp.259-266.

[7] H. Anger, "Scintillation camera," Rev Sci. Inst., vol.29, no.1, pp.27-33, 1958.

[8] Stefan Siegel, Robert W. Silverman, Yiping Shao, Simon R.Cherry, "Simple charge division readout for imaging scintillator arrays using a multi-channel PMT," IEEE Trans. Nucl. Sci., vol. 43, no. 3, pp. 1634$1641,1996$.

[9] Mark B. Williams, Allen R.Goode, victor Galbis-Reig, Stan Majewski, Andrew G. Weisenberger, and Randolph Wojcik, "Performance of a PSPMT based detector for scintimammography," Phys.Med. Biol, 45, pp.781-800, 2000.

[10] Frezghi Habte, Peter D. Olcott, Craig S. Levin, Angela M. Foudray, and Jonathon A. Talcott, " Prototype parallel readout system for position sensitive PNT based gamma ray imaging systems," in Proc. IEEE Nucl. Sci. Symp.Conf. Rec., vol. 4, pp. 1891-1894, 2003.

[11] R. Wojcik, S. Majewski, B. Kross, V. Popov, and A.G. Weisenberger, "Optimized readout of small gamma cameras for high resolution single gamma and positron emission imaging,"2001 IEEE NSS-MIC Conference Record, San Diego, Nov.4-10, 2001.

[12] V. Popov, S. Majewski, A.G.Weisenberger, R. Wojcik, "Analog readout system with charge division type output," in Proc. IEEE Nucl. Sci. Symp. Conf. Rec., vol. 4, pp. 1937-1940, 2001. 